\title{
Development of a biaxial tensile module at synchrotron beamline for the study of mechanical properties of nanostructured films
}

\author{
E. Le Bourhis ${ }^{1}$, B. Girault ${ }^{1}$, P.-O. Renault ${ }^{1}$, P. Goudeau ${ }^{1}$, G. Geandier ${ }^{2}$, D. Thiaudière ${ }^{2}$, R.N. \\ Randriamazaoro ${ }^{3}$, R. Chiron ${ }^{3}$, D. Faurie ${ }^{3}$, O. Castelnau ${ }^{3}$ \\ ${ }^{1}$ LPM-PhyMat, UMR6630 CNRS, Université de Poitiers, B. P. 30179, 86962 Futuroscope, \\ France \\ ${ }^{2}$ Synchrotron SOLEIL, L'Orme des Merisiers, BP 48, 91192 Gif sur Yvette, France \\ ${ }^{3}$ LPMTM, UPR 9001 CNRS, Université Paris-Nord, 93430 Villetaneuse, France
}

\begin{abstract}
We have developed on the DIFFABS-SOLEIL beamline a biaxial tensile machine with synchrotron standard for in-situ diffraction characterization of thin polycrystalline metallic film mechanical response. The machine has been designed to test cruciform substrates coated by the studied film under controlled applied strain field. Technological challenges comprise the fixation of the substrate, the generation of a uniform strain field in the studied (central) volume, the operations from the beamline pilot. Tests on $\mathrm{W}$ and $\mathrm{W} / \mathrm{Cu}$ multilayers films deposited on polyimide substrates are presented.
\end{abstract}

\section{INTRODUCTION}

Understanding the mechanical behavior of nanostructured thin films in relation to their microstructure, in particular to the grain size, is of utmost importance for the development of technological applications [1]. Synchrotron X-ray sources allow for characterizing small volumes of material in an acceptable time schedule. Hence, supported thin films mechanical response has been characterized experimentally in situ by synchrotron X-ray diffraction [2,3] while having in view their microstructure. This is particularly important when the films are multiphased and/or formed by anisotropic elastic crystallites. In such case, when the crystallites are not randomly distributed (for instance in the presence of texture) the film response is expected to be elastically anisotropic. The tests are generally carried out under uniaxial loading, the films being stressed biaxially because of the Poisson ratios mismatch (between the film and substrate). The transversal component of stress is then imposed by the deposited film properties. So far, it is of utmost importance to control both stress components. In that objective we have developed a biaxial loading machine at synchrotron standard allowing for applying in plane normal forces to coated substrates. Experiments on the elastic deformation of isotropic W and on the confined plasticity and fracture of $\mathrm{W} / \mathrm{Cu}$ multilayers are presented.

\section{BIAXIAL TENSILE MACHINE DESIGN}

The tensile machine has been designed to allow for loading along two normal axis cruciform substrates coated by the studied films. The device is to be operated on a beamline at SOLEIL standard (French synchrotron at Gif sur Yvette, France). A micrograph taken at 
DIFFABS beamline and a schematic representation of the machine are shown in Figure 1. The machine is compact $3.5 \mathrm{~kg}$ in weight, $19 \times 19 \times 8.5 \mathrm{~cm}^{3}$ in size with an empty centre and is designed to allow X-ray diffraction under small angles (no shadowing edges). Two couples of motors and force sensors are fixed to the device frame. The 4 motors can be actuated separately in order to keep the studied area at a fixed position in the goniometer (same studied volume, Figure 1). All force sensors were calibrated using dead weights. The cruciform substrates were coated at their centre only and gripped by a cam rotating in a cylinder fixation. A polymeric substrate is chosen to minimize its mechanical contribution as compared to that of the films. Here, we used $125 \mu \mathrm{m}$ thick polyimide (sofimide ${ }^{\circledR}$ from Micel and Kapton $₫$ from Du Pont de Nemours).
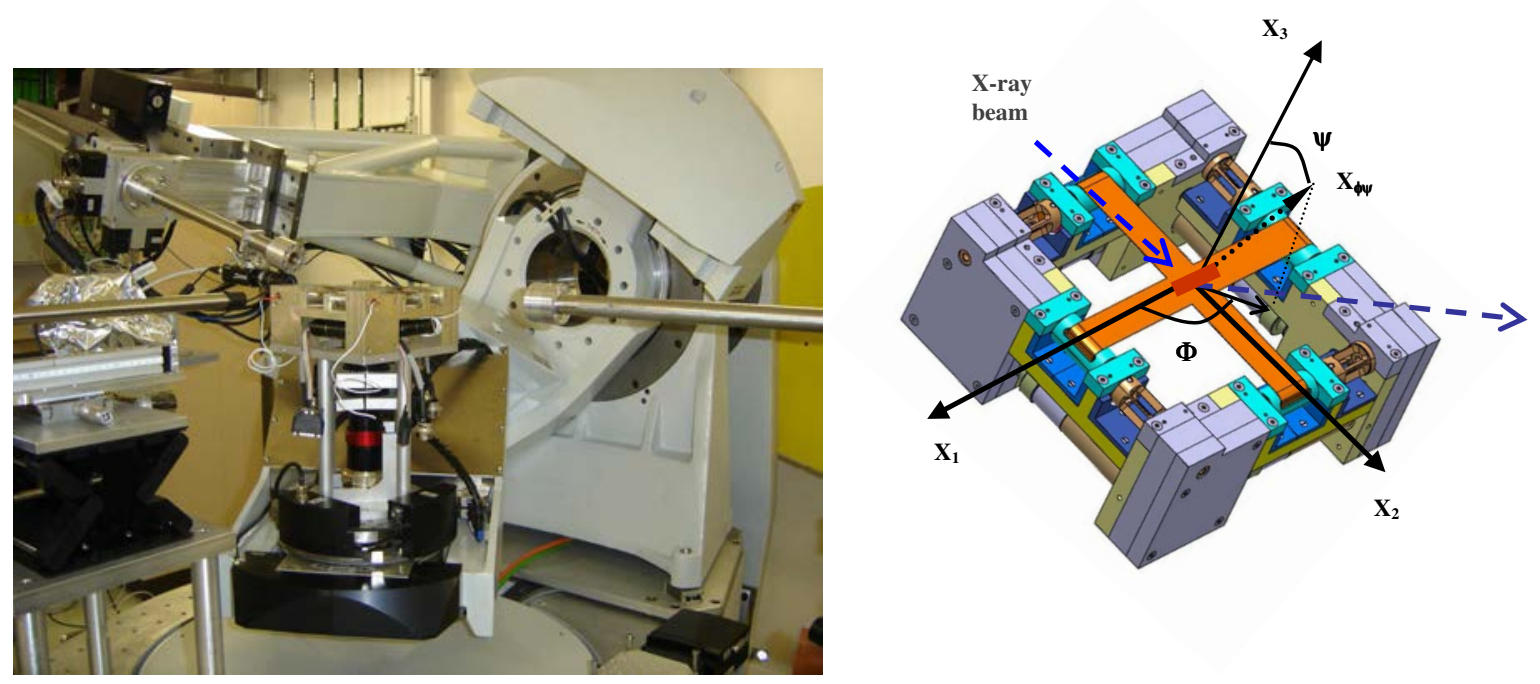

Figure 1 Biaxial tensile device set on DIFFABS-SOLEIL beamline (left) and schematic representation of the biaxial machine, showing the cruciform substrate, gripped by 4 cylinders connected to force sensors and rotated by step motors (right).

One of the most challenging aspects in biaxial testings is the specimen design [4,5]. To perform a biaxial test on sheet material, a cross-shaped specimen is typically used, i.e. cruciform specimen. The objective of the present study was to check that we could perform in situ X-ray strain measurements with high accuracy. Hence, the specimen design was governed by the following constraint: the centre of the substrate specimen must exhibit a homogeneous strain area larger than the irradiated area i.e. a few $\mathrm{mm}^{2}$. Using finite elements analysis (CASTEM code from CEA), we checked that under a $100 \mathrm{~N}$ equibiaxial loading, a uniform strain (deviation less than $1 \%$ ) was generated in a central area of $6 \mathrm{~mm}$ in radius of the cruciform substrate with $20 \mathrm{~mm}$ in width branches and $5 \mathrm{~mm}$ toe weld (see Fig. 2) while the used X-ray beam section at sample surface was about $1 \times 0.3 \mathrm{~mm}^{2}$. It is to be noted that this calculation could be obtained with a 2 dimensional approach in the case of a bare substrate. The actual tested specimens are more complex being coated and require a 3 dimensional approach. In the next section, the measurements are carried out for a coated substrate in order to extract thin film elastic strains and check the biaxiality and uniformity of the loading.

An optical microscope is fixed underneath the machine and allows capturing the bottom surface of the specimens at each load step. Digital image correlation was then used to extract the 
macroscopic strain of the substrate and to check the uniformity of the strain field. In the future, we could pilot the machine in optical macroscopic central strain. Once the coated substrate is set and gripped in the machine, the test is carried out and controlled in deformation imposed by the motor by incremental steps. The forces are measured at each branch. All electronic signals have been set to SOLEIL standards in order to facilitate the experiments.
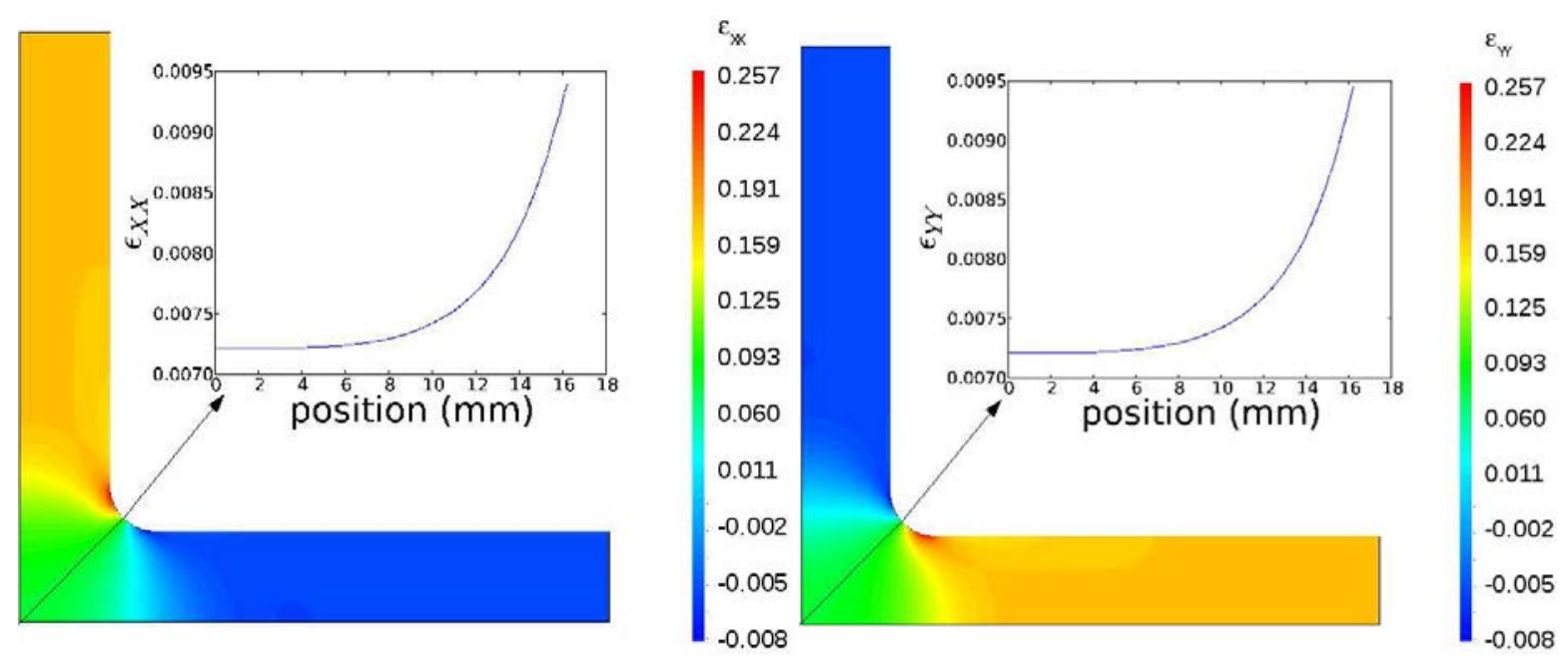

Figure 2 Finite element analysis (FEA) of the strain field in a cruciform substrate loaded under equibiaxial forces of $100 \mathrm{~N}, \varepsilon_{\mathrm{xx}}$ (left) and $\varepsilon_{\mathrm{yy}}$ (right) components. Insets show the respective strain profile along the line shown on the cruciform.

\section{EXPERIMENTAL RESULTS AND DISCUSSION}

\section{Elastic deformation of isotropic W}

For the preliminary tests we used $\mathrm{W}$ that is elastically isotropic. So far, the Poisson ratio of $\mathrm{W}$ is 0.26 (0.284 for the bulk [6]) while we measured 0.37 for the polyimide substrate. These values correspond to a relative Poisson ratio mismatch of 30 and $23 \%$ respectively. As discussed above, an uniaxial loading of coated specimens yield both axial and transversal stress components. Instead, the machine was used to impose an equibiaxial stress field to the coated substrate, except for T6 loading state (Fig. 3). T1 to T5 correspond to equibiaxial forces of 10, $37,63,81$ and $42 \mathrm{~N}$ respectively while $\mathrm{T} 6$ corresponds to non equibiaxial forces of 72 and $48 \mathrm{~N}$ along $\Phi=0$ and $90^{\circ}$ respectively. The $\mathrm{W}$ thin films were produced at room temperature by physical vapor deposition (PVD) with an Ar+-ion-gun sputtering beam at $1.2 \mathrm{keV}$ (Kaufman ion source) in a NORDIKO-3000 system. The base pressure of the deposition chamber was $7 \times 10^{-5}$ $\mathrm{Pa}$ while the working pressure during films growth was approximately $10^{-2} \mathrm{~Pa}$ (For more details see Ref. [3]).

In the case of isotropic materials such as $\mathrm{W}$, the length-scale change is straightforward since the film deforms homogeneously. Measured strains reduce to $[7,8]$

$$
\varepsilon_{\Phi \Psi}^{33}=\left(\frac{1+v_{f}}{E_{f}}\right) \cdot\left(\sigma_{11}^{f} \cos ^{2} \Phi+\sigma_{22}^{f} \sin ^{2} \Phi\right) \cdot \sin ^{2} \Psi-\frac{v_{f}}{E_{f}} \cdot\left(\sigma_{11}^{f}+\sigma_{22}^{f}\right)
$$


$\mathrm{E}_{\mathrm{f}}$ and $v_{\mathrm{f}}$ are the film Young modulus and Poisson ratio respectively, $\sigma_{11}^{f}$ and $\sigma_{22}^{f}$ the principal stress components. $\Phi$ is the rotation angle around surface normal and $\Psi$ the angle between the normal of the diffracting planes and the normal of the specimen surface (Fig. 1). For an equibiaxial stress field $\sigma_{11}^{f}=\sigma_{22}^{f}=\sigma^{f}$, strain is independent of $\Phi$ and writes

$$
\varepsilon_{\Phi \Psi}^{33}=\left(\frac{1+v_{f}}{E_{f}}\right) \cdot\left(\sigma^{f}\right) \cdot \sin ^{2} \Psi-2 \frac{v_{f}}{E_{f}} \cdot\left(\sigma^{f}\right)
$$

We further used two particular angles $\Phi=0$ and $90^{\circ}$ for which strains write

$$
\varepsilon_{0 \Psi}^{33}=\left(\frac{1+v_{f}}{E_{f}}\right) \cdot\left(\sigma_{11}^{f}\right) \cdot \sin ^{2} \Psi-\frac{v_{f}}{E_{f}} \cdot\left(\sigma_{11}^{f}+\sigma_{22}^{f}\right)
$$

and

$$
\varepsilon_{90 \Psi}^{33}=\left(\frac{1+v_{f}}{E_{f}}\right) \cdot\left(\sigma_{22}^{f}\right) \cdot \sin 2 \Psi-\frac{v_{f}}{E_{f}} \cdot\left(\sigma_{11}^{f}+\sigma_{22}^{f}\right)
$$
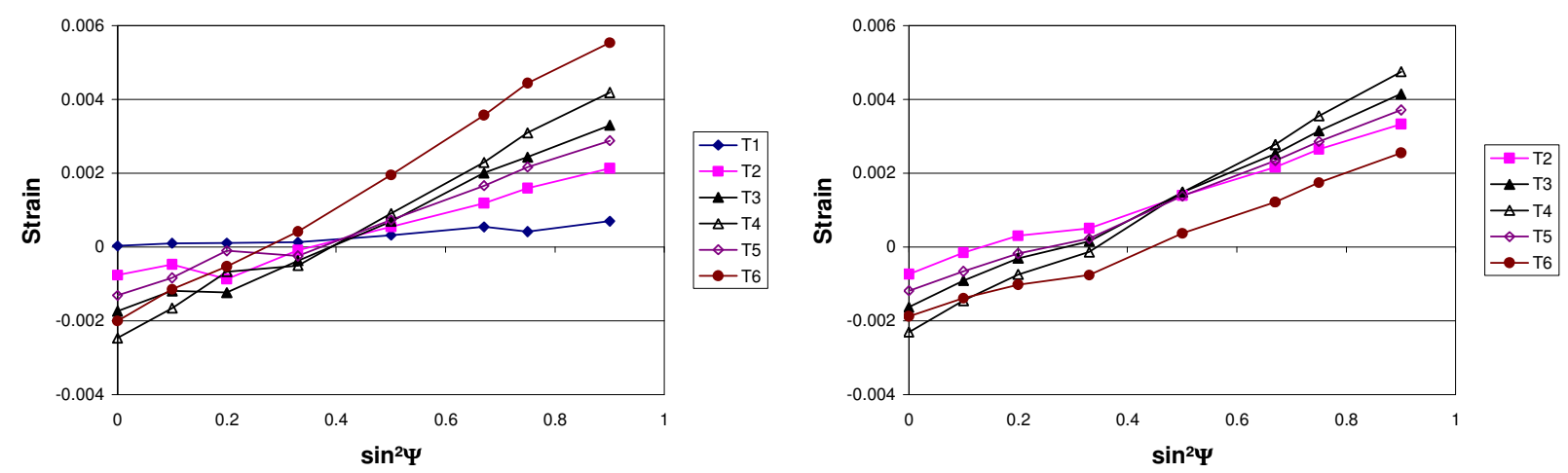

Figure $3(110) \mathrm{W}$ elastic X-ray strains as a function of $\sin ^{2} \Psi$ for $\Phi=0^{\circ}$ ( $\varepsilon_{0 \Psi}^{33}$ left) and $\Phi=90^{\circ}$ ( $\varepsilon_{90 \Psi}^{33}$ right). 6 different loading states TX were used (T1 to T5 being equibiaxial and T6 being non-equibiaxial, see text).

The linear relationship between strain and $\sin ^{2} \psi$ (so called $\varepsilon$ - $\sin ^{2} \psi$ method) is hence established for a single phase elastically isotropic material. So far, most materials show anisotropic elasticity. The mechanical behavior of a polycrystalline thin film is then determined by the distribution of the crystallite orientations within the thin film and the grain interaction $[3,9,10]$. Figure 3 shows the X-ray strains extracted along both axis $\left(\Phi=0\right.$ and $\left.90^{\circ}\right)$ for 6 loading states (T1 to T6) measuring the Bragg peak shifts for different inclinations of the specimen (different angles $\Psi$ ). The strain is calculated using the unloaded state T0 as a reference state: $\varepsilon_{0 \psi}^{33}=\ln \left(\sin \theta_{0 \psi}^{T 0} / \sin \theta_{0 \psi}^{T X}\right)$ where $\theta_{0 \psi}^{T O}$ is the angular position of the considered diffraction peak for the unloaded state and $\theta_{0 \psi}^{T X}$ the corresponding angle for the loaded state TX. As commonly adopted strain is plotted as a function of $\sin ^{2} \Psi$. All curves are linear as expected for an isotropic material [3]. As the load increases the slope of the related curves increases with a loading sequence increasing from T1 to T4 decreasing to T5 while T6 loading state is not equibiaxial. For T2 to T5 loading states, X-ray strains along both directions $\left(\Phi=0\right.$ and $\left.90^{\circ}\right)$ are determined 
in a difference of less than 20\%. Instead for T6 loading state the difference in strain approaches $200 \%$. For equibiaxial stresses Eqs. 1 yield a value of zero strain at $\sin ^{2} \psi=2 v_{f} /\left(1+v_{f}\right) \sim 0.41$ and 0.44 for $v_{\mathrm{f}}$ of 0.26 and 0.284 respectively in good agreement with the obtained experimental values ( 0.42 and 0.46 for $\Phi=0$ and $90^{\circ}$ respectively).

\section{Confined plasticity and fracture of $\mathrm{W} / \mathrm{Cu}$ multilayers}

We further investigated the co-deformation of $\mathrm{W}$ and $\mathrm{Cu}$ sublayers under uniaxial loading. As discussed above a transversal component of stress exists and results from Poisson ratios mismatch. The films were obtained by the same PVD technique, $\mathrm{W}$ and $\mathrm{Cu}$ sublayer thickness being 6 and $18 \mathrm{~nm}$ respectively and with (110) and (111) fiber texture respectively. We took advantage of XRD that is phase selective so that both $\mathrm{W}$ and $\mathrm{Cu}$ elastic strains can be monitored (Fig. 4). We observe that $\mathrm{Cu}$ yields before $\mathrm{W}$ under an applied force of about $5 \mathrm{~N}$ (estimated stress in $\mathrm{Cu}$ sublayer is about $120 \mathrm{MPa}$, using a simple rule of mixture). TEM micrographs (not shown here) indeed reveal dislocations in $\mathrm{Cu}$ sublayer at this step while $\mathrm{W}$ sublayers remain free of dislocations (no dislocation detected).

On increasing the applied force to $8 \mathrm{~N}$, W sublayers yield, plastic deformation continuing until micro-fracture happens in $\mathrm{W}$ sublayers (strain rate being extremely low and about $10^{-8} \mathrm{~s}^{-1}$ ). After increasing further the force, macro-fracture runs through the complete multilayer. The TEM cross-sectional view shown in Fig. 4 was obtained at this step and reveals that fracture happens in mode II (slide mode [11]). From the above observations, we suggest that the confined plasticity regime in $\mathrm{Cu}$ leads to the accumulation of dislocations at $\mathrm{Cu} / \mathrm{W}$ interfaces. Next, $\mathrm{W}$ sublayers deform with $\mathrm{Cu}$ sublayers in planes where Schmid factor maximizes (that is at $\sim 45^{\circ}$ from load axis) when fracture of the films happens in mode II.

It is worth noting that the overall process yields compressive stresses in both $\mathrm{W}$ and $\mathrm{Cu}$ sublayers. This final stress state results from the difference between the substrate and film elastic limit. Indeed, the polyimide substrate remains elastic during the test and unloads completely compressing the $\mathrm{W}$ and $\mathrm{Cu}$ sublayers that plastically deformed.
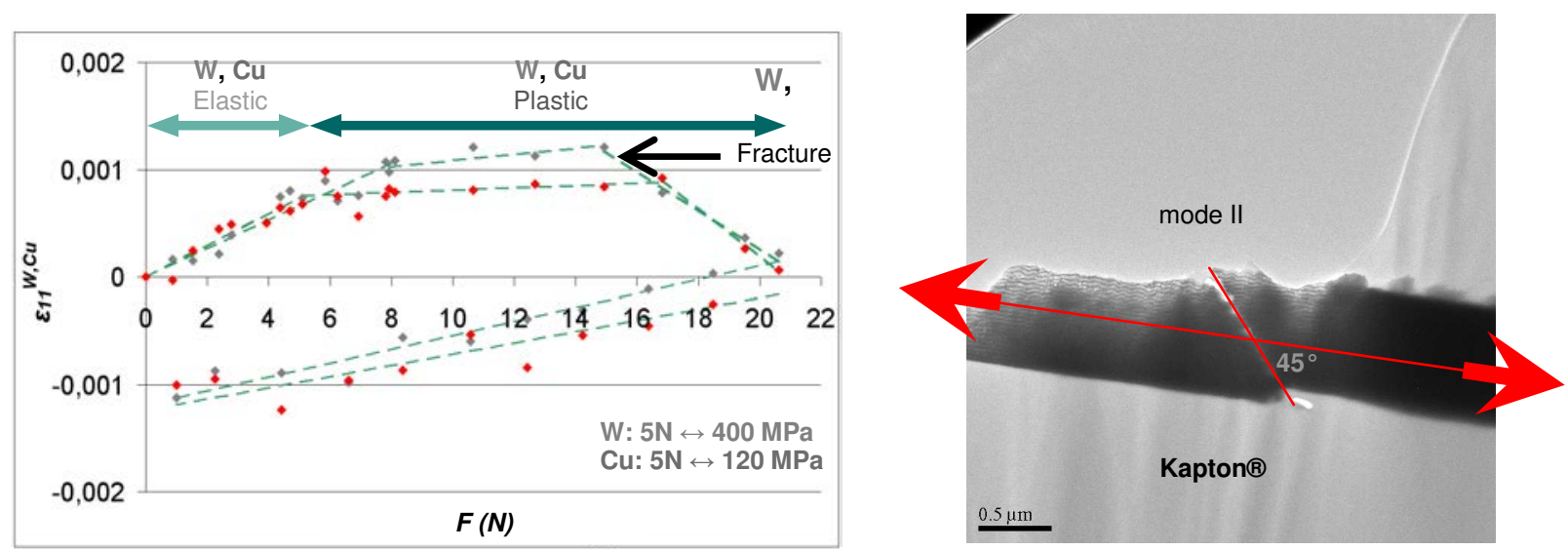

Figure $4 \mathrm{~W}$ and $\mathrm{Cu}$ X-ray elastic strains as a function of Force $\mathrm{F}$ (left) and TEM cross-sectional view after fracture (right). 


\section{CONCLUSIONS}

We have developed on the DIFFABS-SOLEIL beamline a biaxial tensile machine with synchrotron standard for in-situ diffraction characterization of thin polycrystalline metallic film mechanical response. Preliminary tests on $\mathrm{W}$ films deposited on polyimide cruciform substrates show that the device allows for setting a uniform strain field in the characterized volume of a film having a Poisson ratio mismatch with respect to the supporting substrate. Such instrument combined with XRD allows for studying the co-deformation of nanostructured materials like W/Cu multilayers. Further developments are aimed at developing dynamic testing controlled by a ramp of optical strain (determined at substrate backside surface).

\section{ACKNOWLEDGMENTS}

Part of this work has been developed in the ANR project entitled Cmonano (ANR-05NANO-069-03).

\section{REFERENCES}

1. M.A. Meyers, A. Mishra, D.J. Benson, Prog. Mater. Sci. 51 (2006) 427.

2. J. Böhm, P. Gruber, R. Spolenak, A. Stierle, A. Wanner, E. Arzt, Rev. Sci. Inst. 75 (2004) 1110 .

3. D. Faurie, P.-O. Renault, E. Le Bourhis, P. Villain, Ph. Goudeau, and K. F. Badawi, Thin Solid Films, 469-470 (2004) 201.

4. A. Hannon, and P. Tiernan, J. Mater. Proc. Techn. 198, 1 (2008).

5. S. Demmerle, J.P. Boehler, J. Mech. Phys. Solids 41, 143 (1993)

6. P.O. Renault, K.F. Badawi, L. Bimbault, P. Goudeau, E. Elkaïm, J.P. Lauriat, Appl. Phys. Lett. 73 (1998) 1953

7. V. Hauk (1997), Structural and residual stress analysis by non destructive methods:

Evaluation, application, assessment. Elsevier Science, Amsterdam.

8. I.C. Noyan, J.B. Cohen (1987) Residual stresses. Measurements by diffraction and interpretation, Springer Verlag, New York.

9. S. Matthies, H. G. Priesmeyer, M. R. Daymond, J. Appl. Cryst. 34, 585 (2001).

10. D. Faurie, O. Castelnau, R. Brenner, P.-O. Renault, E. Le Bourhis, Ph. Goudeau, J. Appl. Cryst. 42 (2009) doi:10.1107/S0021889809037376.

11. E. Le Bourhis (2008), Glass mechanics and technology, Wiley, Weinheim. 